\title{
ON THE DECOMPOSITION OF STOCHASTIC DISCOUNTED CASH FLOWS (*)
}

\author{
F. BECCACECE \\ Istituto di Metodi Quantitativi \\ Universita Bocconi

\section{LI CALZI} \\ Graduate School of Business \\ Stanford University and \\ Istituto di Matematica Finanziaria \\ Universild di Torino
}

Versione definitiva pervenuta il 25/10/1991

This paper extends previous results by Peccati [7] and Beccacece [1] on the decomposition of the discounted cash flow for deterministic financial operations to the stochastic case. Modelling financial operations as processes whose cumulant is a semimartingale, we obtain a very general decomposition formula which allows one to consider even random discount factors.

\section{Introduction}

Recently, a more general methodology for the evaluation of financial operations has been proposed by Peccati [7]. In essence, its aim is to integrate the traditional criterion based on the discounted cash flow with the kind of information provided by an analysis based on the internal rate of return. Due to the ensuing synergy of information, the new methodology has proved quite effective both as a general approach to the evaluation of financial operations and as a more refined tool for practical analysis.

As developed by Peccati and his collaborators (see [9] and the bibliography therein contained), such methodology centers on the existence of a decomposition formula for the discounted cash flow, which makes the period contributions explicit by referring them to a generalized notion of intemal rate of return. With the partial exception of [6], however, the literature has so far dealt only with deterministic cash flows. At least in part, such lack of attention to the more realistic case of stochastic cash flows can be imputed to the absence of a corresponding decomposition formula. The purpose of this paper is to provide such a formula in a very general setting, which may be used to unify the approach to deterministic and stochastic cases.

(*) This work has been partially supported by C.N.R. and M.U.R.S.T. One of the authors wishes to thank M. Armott for drawing (and keeping) us into this project. 
The setting we use centers on the notion of a (random) standard financial operation. We introduce such concept to generalize the definition of a deterministic financial operation given in [2] so that most ot the recent literature on the modelling of stochastic cash flows can be easily taken into account. Despite the extent of the resulting class of standard financial operations, we find that a simple unified treatment is made possible by relying on the theory of stochastic calculus.

The paper is organized as follows. After recalling a few definitions from the theory of stochastic calculus in Section 1.1, in Section 2 we introduce the definition of standard financial operation and accordingly generalize the concepts of internal financial law and outstanding, which are crucial to the decomposition method. Building on these notions, we obtain a general decomposition formula for standard financial operations for the case of deterministic discount factors in Section 3.1. The whole approach is then extended in Section 3.2 to the case where the discount factors involved are random. To illustrate the applicability of the concepts introduced, we carry on an example drawn by the literature on risk theory.

\subsection{Preliminaries form stochastic calculus}

We recall a few definitions form the theory of stochastic calculus. For a more complete treatment see Protter [10], whose notation is followed here. Let $\left(\Omega, \mathcal{F}, P,\left\{\mathcal{F}_{t}\right\}\right.$ ) be a filtered complete probability space satisfying the usual conditions (see [10]). Given a stochastic process $X$ on $(\Omega, \mathcal{F}, P)$ we write $X_{t}$ instead of $X(t, \omega)$ and $X_{t-}$ for $\lim _{s \uparrow t} X(t, \omega)$. Moreover, we define $\Delta X_{t}=X_{t}-X_{t-}$ to be the jump at $t$. Finally, we set $X_{0-}=0$ by convention; remark however that we do not require $X_{0}=0$.

A stochastic process $X$ is adapted if $X_{t}$ is $\mathcal{F}_{t}$-measurable for all $t \geq 0$ and it is càdlàg if it a.s. has right continuous sample paths with left limits. An adapted càdlàg process $M$ is a local martingale if there exists a sequence of increasing stopping times $\left\{T_{n}\right\}$ such that $\lim _{n \rightarrow+\infty} T_{n}=\infty$ a.s. and $X_{t \wedge T_{n}} 1_{\left\{T_{n}>0\right\}}$ is a uniformly integrable martingale for each $n$. An adapted càdlàg process $V$ is a finite variation process if almost all of its paths are of finite variation on each compact interval of $\mathbf{R}^{+}$.

An adapted càdlàg process $X$ is a semimartingale if it can be written $X=M+V$. where $M$ is a local martingale with bounded jumps and $V$ is a finite variation process. Examples of semimartingales include Brownian motions, Lévy processes, square integrable martingales with càdlàg paths, supermartingales and finite variation processes.

Given a semimartingale $X$ and an adapted left continuous process $H$ with right limits, it is possible to consistently define another stochastic process $J_{X}(H)=\int H_{\mathbf{a}} \mathbf{d} X$, which is called the stochastic integral of $H$ with respect to the integrator $X$. When evaluated at $t$, we denote this process by

$$
\left[\int H_{s} \mathrm{~d} X_{\mathrm{a}}\right]_{t}=\int_{0}^{t} H_{\mathrm{o}} \mathrm{d} X_{\mathrm{s}}=\int_{[0, t]} H_{\mathrm{s}} \mathrm{d} X_{\mathrm{s}} .
$$


Whenever 0 is to be excluded from the integral, we use the notation

$$
\int_{0^{+}}^{t} H_{s} \mathrm{~d} X_{s}=\int_{(0, t]} H_{s} \mathrm{~d} X_{\mathrm{s}} .
$$

The stochastic integral process preserves most of the crucial properties of the standard Lebesgue integral. Skipping the details of its construction (for which see [10]), we will only say that given two semimartingales $X$ and $Y$ the integral of $Y_{-}$with respect to $X$ always exists and it is also a semimartingale.

Given two semimartingales $X$ and $Y$, we define the quadratic covariation of $X, Y$ as the stochastic process given by

$$
[X, Y]=X Y-\int X_{-} \mathrm{d} Y-\int Y_{-} \mathrm{d} X
$$

and denote by $[X, Y]^{c}$ its path by path continuous part. Then, we say that a semimartingale $X$ is quadratic pure jump if $[X, X]^{c}=0$. A simple sufficient condition for a semimartingale $X$ to be quadratic pure jump is that $X$ has paths of finite variation on compacts. Thus, for instance, any Poisson process is quadratic pure jump.

An important result that we will need later links the theory of stochastic differential equations to semimartingales. We present it an a less general form more suited to our purposes. A function $f: \mathbf{R}^{+} \times \mathbb{R}^{n} \rightarrow \mathbb{R}$ is said to be Lipschitz if there exists a (finite) constant $k$ such that $|f(t, x)-f(t, y)| \leq k|| x-y \|$, for all $t \in \mathbf{R}^{+}$; and it is said to be regular if it is right-continuous with left limits in $t$ for all $x \in \mathbf{R}^{n}$.

THEOREM 1. Let $A=\left(A^{1}, \ldots, A^{n}\right)$ and $Z=\left(Z^{1}, \ldots, Z^{m}\right)$ be two vectors of semimartingales, with $Z_{0}=0$. Assume that $f_{j}^{i}$ are all regular Lipschitz functions, for $i=1, \ldots, n$ and $j=1, \ldots, m$. Then, the system of equations

$$
X_{t}^{i}=A_{t}^{i}+\sum_{j=1}^{m} \int_{0}^{t} f_{j}^{i}\left(s, X_{s-}\right) \mathrm{d} Z_{s}^{j} \quad i=1, \ldots, n
$$

has a unique solution $X$ in the space of the vectors of adapted càdlàg processes and such solution is a semimartingale.

\section{The basic model}

\subsection{Standard financial operations}

Given a generic financial operation, we can describe the temporal evolution of its cash flow by a function $A: \mathbf{R}^{+} \times \Omega \rightarrow \mathbf{R}$ which gives at any time $t \in \mathbf{R}^{+}$the current (undiscounted) cumulative net flow from the operation. We call such function the cumulant of the operation. In the sequel, we will often identify a financial operation with its cumulant. Throughout this paper, moreover, we will restrict attention to the subclass of stochastic financial operations whose cumulants are semimartingales. 

semimartingale.

This definition has a fairly innitive interpretation. Indeed, recall that every semimartingale can be decomposed into a finite variation process $M$ and a local martingale with bounded jumps $V$. Thus, a standard financial operation is the sum of the two random cumulants $M$ and $V$. The finite variation process $M$ extends to the stochastic case the assumption of finite variation which has been long recognized as the minimal regularity condition to be required from a deterministic financial operation (see [2]). The local martingale $V$ represents instead unpredictable stochastic fluctuations in the cumulative net flow.

In general, both $M$ and $V$ are random processes and thus they are undistinguishable in practice. However, if we make the further assumption that $M$ is deterministic, the intuitive interpretation of $V$ as an unpredictable fluctuation of the cumulant becomes even more natural. In fact, suppose that there is a given deterministic cumulative net flow $M$ additively affected by some underlying source of uncertainty $V$. The agent may receive information over time about $V$, and such arrival of information is modelled as a filtration. Apart from minor technicalities, the assumption that $V$ is a local martingale implies that, given the available information at time $s$, the best guess as to what will be the net cumulative cash flow at some time $t>s$ is given by $M_{t}+V_{s}$, i.e. by the sum of the deterministic cumulant and the current value of $V$.

For instance, consider the following classical example drawn from risk theory (see [3]). An insurance company with initial capital $u$ intakes premia at a rate of $c$ per unit of time; in exchange for this, it must be ready to pay a stochastic amount of money each time that a claim is received. Claims arrive according to a point process $N=\{N(t) ; t \geq 0\}$ with unit increments and are modelled as a sequence of independent and identically distributed random variables $\left\{Z_{k}\right\}$. The risk process $\rho$ faced by the company is defined by

$$
\rho(t)=u+c t-\sum_{k=1}^{N(t)} Z_{k}
$$

and it is immediately seen that $\rho$ is a standard financial operation where $M_{t}=u+$ ct is the deterministic cumulant and $V_{t}=-\sum_{k=1}^{N(t)} Z_{k}$ is the unpredictable stochastic disturbance.

It is important to remark that the plausibility of the interpretation just provided is not crucial. The main importance of the class of standard financial operations is that it encompasses almost all the models of financial operations usually encountered in the current literature. As of the deterministic cumulants considered by the traditional mathematics of finance, they all enjoy the finite variation property and thus the definition of a 
standard financial operation subsumes them for $V=0$. As of the stochastic cumulants considered in the modern mathematics of finance, they are usually obtained as solutions of stochastic differential equations which, under assumptions even milder than those of Theorem 1 , are nothing but semimartingales.

Finally, we mention that it can be shown that the set of standard financial operations is an algebra. In particular, therefore, we can take linear combinations of standard financial operations to obtain other financial operations which are still standard. Hence, such class is closed with respect to the usual composition operators for financial cash flows.

\subsection{DCF and outstanding}

Continuing with our example, we notice that traditional risk theory is interested in determining the probability of ruin faced by the insurance company for a given risk process. We wish to consider a different viewpoint and look at risk processes as altemative investment opportunities open to an insurance company. In fact, when starting a new policy, the company may vary deductibles or other conditions so as to generate different risk processes. If we regard each risk process as a standard financial operation generating a random cash flow, the company faces the problem of choosing the best insurance policy according to some criterion. We provide in the following the mathematics necessary to apply the methodology of the decomposition of discounted cash flows when the financial operations under exam are stochastic.

To this purpose, we need to introduce the notions of discounted cash flow (for short, DCF) and outstanding for a standard financial operation. Unless explicitly noted, it is assumed throughout the rest of this work that all the financial operations are standard and take place in the time interval $[0, T], T<+\infty$. While the choice of 0 as left boundary point is only a convenient nomalization, the assumption of a bounded time interval is a crucial simplification. Relaxing such assumption to allow for an unbounded time horizon can be done only at the price of some heavy-duty technical assumptions that do not seem to add any special insight.

We recall first a few definitions. A function $\mu: \mathbf{R}^{+} \rightarrow[0,1]$ is said to be a discount factor if it is nonincreasing and $\mu_{0}=1$. For the moment, we will restrict attention to the class $\Delta$ of (deterministic) discount factors which are continuously differentiable and strictly positive. The first assumption is technical and will be weakened to finite variation in Section 3.2. The second one, instead, is merely simplifying in that it allows for the discount factors in the class $\Delta$ the elegant representation $\mu=e^{-m}: \mathbf{R}^{+} \rightarrow[0,1]$, where $m: \mathbf{R}^{+} \rightarrow \mathbf{R}^{+}$is a nondecreasing continuously differentiable function such that $m_{0}=0$.

DEFINITION 2. Let $A$ be a standard financial operation and $\mu=e^{-m}$ a discount factor 


$$
G(\mu)=A_{0}+\int_{0}^{T} \mu_{s} \mathrm{~d} A_{s}=A_{0}+\int_{0}^{T} e^{-m_{s}} \mathrm{~d} A_{s} .
$$

This definition is a straightforward generalization of the traditional one for deterministic financial operations. According to it, the process defining the DCF evolves as a semimartingale whose value at $T$ determines the discounted cash flow of the operation. For instance, if we define $\tau_{k}=\inf \{t: N(t)=k\}$ to be the waiting time for the $k$-th claim in our example, (2) provides the following natural expression for the (random) discounted cash flow of the risk process (1):

$$
G(\mu)=u+c \int_{0}^{T} e^{-m_{0}} \mathrm{~d} s-\sum_{k=1}^{N(T)} Z_{k} e^{-T_{k}} .
$$

In general, the DCF of a standard financial operation is a random variable which need not to be square integrable. Since it is not necessary to our development, however, we will not discuss any condition ensuring the square integrability of the DCF except to mention that it essentially requires a particular norm of $A$ to be finite and that this will happen in most cases of practical relevance.

We now move to consider the outstanding of a stochastic financial operation. First, we recall a few definitions from the theory of deterministic financial operations. The notion of internal financial law generalizes that one of internal rate of retum by removing the restriction that the discount law annihilating the DCF be exponential. Given a deterministic financial operation $A$, we say that the discount factor $\mu$ is an internal financial law (for short, IFL) if $G(\mu)=0$. For a stochastic financial operation, the DCF is a random variable and thus the definition of an IFL has to be generalized accordingly. A natural proposal, first advanced in Luciano and Peccati [6], is the following.

DEFINITION 3. For a standard financial operation $A$, we say that $\nu \in \Delta$ is an internal financial law in mean if $E[G(\nu)]$ exists and it is 0 .

As it is the case for a deterministic financial operation, a standard financial operation $A$ may admit in general several IFL's in mean. In the following we assume that all the standard financial operations considered admit at least one IFL in mean, denoted by $\nu=e^{-n} \in \Delta$.

Another important concept is that one of outstanding, which generalizes to a generic financial operation the notions of outstanding debt for a financing operation and of exposure for an investment (see [8]). Its origin can be traced back to the depreciation formula derived by Hotelling in 1925 (see [4]). We provide its definition for the general case of 
a standard financial operation; under the further assumption that the operation is deterministic, one can easily recover the traditional definition (see [1] for a derivation of this latter one).

DEFINITION 4. Let $A$ be a standard financial operation. If $\nu=e^{-n} \in \Delta$ is an IFL in mean for $A$, we define the outstanding $W$ of $A$ with respect to $\nu$ to be the unique adapted càdlàg process which solves the equation

$$
W_{t}=A_{t}-\int_{0}^{t} W_{-s} \mathrm{~d} \log \nu_{s}=-A_{t}+\int_{0}^{t} n_{s}^{\prime} W_{s-} \mathrm{d} s
$$

Remark that since $\nu=e^{-n}$ and thus $n$ are continuously differentiable, $n^{\prime}$ is bounded on the compact interval $[0, T]$. Therefore, $f(t, x)=n_{t}^{\prime} x$ is regular Lipschitz. Hence, by Theorem 1 , such equation has a unique solution in the space of adpted càdlàg processes which is also a semimartingale. In other words, if $\nu$ is an IFL in mean for the standard financial operation $A$, the construction of the outstanding yields another uniquely defined standard financial operation. Finally, we notice that $W_{0}=-A_{0}$.

The definition just given obtains the outstanding as the solution of a stochastic exponential equation with an exogeneous driving term. Exploiting this simple observation and that part of the regularity assumption stating that $\nu$ is continuous, we can apply Theorem 5.52 from [10] to conclude that the following closed form expression for the outstanding $W$ of $A$ holds:

$$
W_{\mathrm{t}}=-\frac{1}{\nu_{\mathrm{t}}}\left\{A_{0}+\int_{0^{+}}^{\mathrm{t}} \nu_{\mathrm{s}} \mathrm{d} A_{\mathrm{s}}\right\} \text {; }
$$

Rewritten as

$$
\nu_{t} W_{\mathrm{t}}=-\left\{A_{0}+\int_{0}^{\mathrm{t}} \nu_{\mathrm{s}} \mathrm{d} A_{\mathrm{s}}\right\}
$$

such expression provides immediately a natural interpretation of the outstanding at time $t$ as the backward evaluation (see [5]) of the cash flow of a financial operation.

If we assume for notational simplicity the exponential intemal financial law $\nu_{t}=$ $e^{-r},(4)$ and a bit of algebra applied to our example give, conforming to our intuition,

$$
W_{t}=-u e^{r t}-c \int_{0}^{t} e^{n(t-s)} \mathrm{d} s+\sum_{k=1}^{N(t)} Z_{k} e^{n\left(t-r_{k}\right)} .
$$




\section{Decomposition formulas}

In this section we state some general decomposition formulas for the DCF of a standard financial operation. We discuss first an intermediate case where the discount factors are assumed to be deterministic, and then generalize the result obtained to the case of random discount factors.

\subsection{Deterministic discount factors}

Before giving the intermediate decomposition formula for the case of deterministic discount factors, we need to state a useful result whose proof is omitted because it can be easily obtained as a particular case of Lemma 4.

LEMMA 2. Let $\mu \in \Delta$ and $W$ a standard financial operation. Then the process $\mu W$ is also a standard financial operation. Moreover,

$$
\mu_{\mathrm{t}} W_{\mathrm{t}}-\mu_{0} W_{0}=\int_{0}^{t} W_{\mathrm{o}-} \mathrm{d} \mu_{\mathrm{s}}+\int_{0}^{t} \mu_{\mathrm{s}} \mathrm{d} W_{\mathrm{s}}
$$

Using such lemma, the decomposition formula for deterministic discount factors is established as follows.

PROPOSITTON 3. Lct $A$ be a standard financial operation and $\mu=e^{-m} \in \Delta$. If $\nu=e^{-n} \in \Delta$ is an IFL in mean for $A$, let $W$ be the outstanding of $A$ with respect to $\nu$. Then

$$
G(\mu)=-\mu_{T} W_{T}+\int_{0}^{T}\left(n_{s}^{\prime}-m_{s}^{\prime}\right) \mu_{d} W_{s} \mathrm{~d} s .
$$

Proof. By definition of outstanding,

$$
\int_{0}^{T} \mu_{s} \mathrm{~d} W_{s}=-\int_{0}^{T} \mu_{\mathrm{d}} \mathrm{d} A_{\mathrm{s}}+\int_{0}^{T} r_{s}^{\prime} \mu_{s} W_{s-} \mathrm{d} s .
$$

On the other hand, recall from the discussion at the end of Section 2.2 that the outstanding $W$ is also a standard financial operation. Thus, interpreting Lemma 2 as an integration by pars formula, we have

$$
\int_{0}^{T} \mu_{\mathrm{o}} \mathrm{d} W_{s}=\mu_{T} W_{T}-\mu_{0} W_{0}-\int_{0}^{T} W_{s-} \mathrm{d} \mu_{s}=\mu_{T} W_{T}+A_{0}+\int_{0}^{T} m_{s}^{\prime} \mu_{s} W_{s-} \mathrm{d} s .
$$

Equating the right-hand sides, we obtain

$$
A_{0}+\int_{0}^{T} \mu_{\mathrm{s}} \mathrm{d} A_{\mathrm{s}}=-\mu_{T} W_{T}+\int_{0}^{T}\left(n_{\mathrm{s}}^{\prime}-m_{\mathrm{s}}^{\prime}\right) \mu_{\mathrm{s}} W_{\mathrm{s}} \mathrm{d} s
$$


and (6) is established.

Continuing with our example, let us obtain directly the decomposition of the discounted cash flow given by (6). For simplicity, we assume that both the intemal financial law $\nu_{t}=e^{-n t}$ and the discount factor $\mu_{t}=e^{-m t}$ are exponential. Starting from (3), we have:

$$
\begin{aligned}
G(\mu) & =u+c \int_{0}^{T} e^{-m s} \mathrm{~d} s-\sum_{k=1}^{N(T)} Z_{k} e^{-m \tau_{k}} \\
& =u+c\left(\frac{1-e^{-m T}}{m}\right)-\sum_{k=1}^{N(T)} Z_{k} e^{-m \tau_{k}} .
\end{aligned}
$$

Considering separately the three components of the sum, we find respectively

$$
\begin{aligned}
u & =u\left[e^{(n-m) T}-(n-m) \int_{0}^{T} e^{(n-m) s} \mathrm{~d} s\right] \\
c\left(\frac{1-e^{-m T}}{m}\right) & =c\left[\frac{e^{(n-m) T}-e^{-m T}}{n}-\frac{n-m}{n} \int_{0}^{T}\left(e^{(n-m) s}-e^{-m s}\right) \mathrm{d} s\right] \\
\sum_{k=1}^{N(T)} Z_{k} e^{-m \tau_{k}} & =e^{(n-m) T} \sum_{k=1}^{N(T)} Z_{k} e^{-\pi_{k}}-(n-m) \int_{0}^{T}\left[e^{(n-m) s} \sum_{k=1}^{N(s)} Z_{k} e^{-n T_{k}}\right] \mathrm{d} s
\end{aligned}
$$

Substituting back these expressions, we obtain

$$
\begin{aligned}
G(\mu)= & u e^{(n-m) T}+c\left[\frac{e^{(n-m) T}-e^{-m T}}{n}\right]-e^{(n-m) T} \sum_{k=1}^{N(T)} Z_{k} e^{-n \pi_{k}} \\
& -(n-m) \int_{0}^{T}\left[u e^{(n-m) s}+\frac{c}{n}\left(e^{(n-m) s}-e^{-m s}\right)-e^{(n-m) s} \sum_{k=1}^{N(s)} Z_{k} e^{-m T_{k}}\right] \mathrm{d} s \\
= & -e^{-m T} W_{T}+\int_{0}^{T}(n-m) e^{-m s} W_{s} \mathrm{~d} s
\end{aligned}
$$

which is nothing but the decomposition given in (6). The amount of calculations involved in the direct derivation of the decomposition shows the practical importance of Proposition 3.

\subsection{Random discount factors}

The decomposition formula just obtained may be generalized to the case where one allows for stochastic discount factors. Before discussing such version of the decomposition formula, however, we need to introduce the notion of a random discount factor. 
Since a deterministic discount factor $\mu$ is characterized by the property of being nonnegative, nonincreasing and normalized so that $\mu_{0}=1$, it appears natural to maintain at least some of these properties for its stochastic counterpart. This motivates the following definition.

DEFINITION 5. A finite variation process $\rho: \mathbb{R}^{+} \times \Omega \rightarrow[0,1]$ such that $\rho_{0}=1$ is said to be a random discount factor.

Such definition requires a random discount factor to a.s. satisfy pathwise properties weaker than those characterizing a deterministic discount factor. In particular, we allow a random discount factor not to be nonincreasing with respect to $t$. There are two reasons for this: first, there is no difficult in making the stronger assumption that a random discount factor is a.s. nonincreasing. Second, it seems preferable not to impose such restriction when the discount factor arises in a stochastic environment. We remark, on the other hand, that it remains necessary that the pathwise variation of the random discount factor be not too irregular.

It is worth mentioning that the choice of the range $[0,1]$ for the random discount factor is to some extent arbitrary: in fact, it is possible to define it to be $[0,+\infty)$ without affecting any of the conclusions below. As above, we will also make the additional simplifying assumption that the random discount factors we consider are always positive. We denote the class of positive random discount factors by $\bar{\Delta}$. If $\rho \in \bar{\Delta}$, we can write $\rho=e^{-r}$ where $r: \mathbf{R}^{+} \times \Omega \rightarrow \mathbf{R}^{+}$is a finite variation process such that $r_{0}=0$.

Having defined random discount factors, it becomes necessary to reexamine the notions of IFL and outstanding. In fact, while the definition of the discounted cash flow is formally identical to (2) after replacing $\Delta$ by $\bar{\Delta}$ and the integrand $\mu_{s}$ by $\rho_{s-}$, the possibility of randomness in the discount factor allows for an altemative definition of the IFL and thus a different notion of outstanding.

DEFINITION 6. For a standard financial operation $A$, we say that $\nu \in \Delta$ is an internal financial law in mean if $E[G(\nu)]$ exists and it is 0 . We say instead that $\sigma \in \bar{\Delta}$ is a.s. an internal financial law if $G(\sigma)=0$ with probability one.

The two notions coincide for deterministic financial operations. However, while the definition of IFL in mean leads to a deterministic discount factor, an a.s. IFL is in general random. It would be possible to extend the definition of IFL in mean to the class $\bar{\Delta}$ and therefore allow for a random IFL in mean, but this is beyond the scope of this work. We remark that in general a standard financial operation may have several IFL's both in mean and a.s.; moreover, it does not follow neither that an IFL in mean is an a.s. IFL nor viceversa. In the following we assume that all the standard financial operations considered admit at least one IFL in mean and one a.s. IFL, respectively denoted by 
$\nu=e^{-n} \in \Delta$ and $\sigma=e^{-s} \in \bar{\Delta}$.

DEFINITION 7. Let $A$ be a standard financial operation. If $\beta=e^{-b}$ is an IFL (in mean or a.s.) for $A$, we define the outstanding $W$ of $A$ with respect to $\beta$ to be the unique adapted càdlàg process which solves the equation

$$
W_{t}=-A_{t}-\int_{0}^{t} W_{s-} \mathrm{d} \log \beta_{s}=-A_{t}+\int_{0}^{t} W_{s-} \mathrm{d} b_{s} .
$$

Since $\beta \in \bar{\Delta}$, it is a finite variation process and therefore a semimartingale. Moreover, $b_{0}=0$ and $f(t, x)=x$ is regular Lipschitz. Thus, by Theorem 1, Equation (7) has a unique solution in the space of adapted càdlàg processes which is also a semimartingale. Therefore, this definition generalizes Definition 4 and the observations following this latter one hold here as well. In particular, if $\beta$ is also continuous, Theorem 5.52 from [10] applies again and a few simple computations give a closed form expression for $W$ which mirrors (4):

$$
W_{t}=-\frac{1}{\beta_{t}}\left\{A_{0}+\int_{0^{+}}^{t} \beta_{s} \mathrm{~d} A_{s}\right\}
$$

so that the natural interpretation of the outstanding as a backward evaluation is maintained.

The following lemma is the analog of Lemma 2 for random discount factors.

LEMMA 4. Let $\rho \in \bar{\Delta}$ and $W$ a standard financial operation. Then the process $p W$ is also a standard financial operation. Moreover.

$$
\rho_{t} W_{t}-\rho_{0} W_{0}=\int_{0}^{t} W_{s-} \mathrm{d} \rho_{s}+\int_{0}^{t} \rho_{s-} \mathrm{d} W_{s}+\sum_{0<s \leq t} \Delta \rho_{s} \Delta W_{s} .
$$

Proof. The complete proof is standard but long. A quick way to give it is to collate a few results from Protter [10]. Since $\rho$ is a random discount factor, it is a finite variation process and thus by its Theorem 2.26 it is a quadratic pure jump semimartingale. Therefore, by its Theorem 2.28, the quadratic covariation is

$$
[\rho, W]_{t}=\rho_{0} W_{0}+\sum_{0<s \leq t} \Delta \rho_{s} \Delta W_{s}
$$

and the result follows by Corollary 2 to Theorem 2.22 .

Remark that if $\rho$ is continuous the summation term is zero. This establishes Lemma 2.

With respect to the decomposition of the DCF, the most important analytical property of the outstanding associated to an a.s. IFL or to an IFL in mean is the following. 
PROPOSITION 5. Let $A$ be a standard financial operation. If $W$ is its outstanding with respect to the a.s. IFL $\sigma \in \bar{\Delta}$, then

$$
-\sigma_{T} W_{T}+\sum_{0<s \leq T} \Delta \sigma_{s} \Delta W_{s}=0 \quad \text { a.s. }
$$

Similarly, if $W$ is its outstanding with respect to the IFL in mean $\nu \in \Delta$, then

$$
E\left[-\nu_{T} W_{T}+\sum_{0<s \leq T} \Delta \nu_{\mathrm{s}} \Delta W_{\mathrm{o}}\right]=0
$$

Proof. By the definition of a.s. IFL, it holds almost surely that

$$
A_{0}+\int_{0}^{T} \sigma_{s-} \mathrm{d} A_{s}=0
$$

By definition of outstanding,

$$
A_{\mathrm{t}}=-W_{\mathrm{t}}+\int_{0}^{\mathrm{t}} W_{\mathrm{s}-\mathrm{d}} \mathrm{d} s_{\mathrm{s}} .
$$

Substituting in (10), we obtain

$$
A_{0}-\int_{0}^{T} \sigma_{s-} \mathrm{d} W_{s}+\int_{0}^{T} \sigma_{s-} W_{s-} \mathrm{d} s_{s}=0,
$$

which by Lemma 4 can be rewritten as

$$
A_{0}-\sigma_{T} W_{T}+\sigma_{0} W_{0}+\int_{0}^{T} W_{s-d} \mathrm{~d} \sigma_{s}+\sum_{0<s \leq T} \Delta \sigma_{s} \Delta W_{s}+\int_{0}^{T} \sigma_{s-} W_{s-} \mathrm{d} s_{s}=0 .
$$

Thus, given the initial conditions $\sigma_{0}=1$ and $W_{0}=-A_{0}$, it suffices to remark that

$$
\int_{0}^{T} W_{s-} d \sigma_{s}=-\int_{0}^{T} \sigma_{s-} W_{s-} d s_{s}
$$

and the result is established.

The proof of the second part of the proposition is analogous.

Remark that in particular Equation (9) holds if $W$ is the outstanding of $A$ with respect to the a.s. IFL $\sigma$.

We are now ready to give the general decomposition formula for random discount factors. 
PROPOSITION 6. Let $A$ be a standard financial operation and $\rho=e^{-r} \in \bar{\Delta}$. If $\beta=e^{-b}$ is an IFL (either in mean or a.s.) for $A$, let $W$ be the outstanding of $A$ with respect to $\beta$. Then

$$
G(\rho)=-\rho_{T} W_{T}+\int_{0}^{T} \rho_{s-} W_{s-} \mathrm{d}\left(b_{\mathrm{s}}-r_{\mathrm{s}}\right)+\sum_{0<s \leq T} \Delta \rho_{\mathrm{s}} \Delta W_{\mathrm{s}}
$$

In particular, if $\beta=\sigma$ is an a.S. IFL, this reduces to

$$
G(\rho)=\int_{0}^{T} \rho_{s-} W_{s-} \mathrm{d}\left(s_{s}-\tau_{s}\right)
$$

Moreover, in both cases it follows that

$$
E[G(\rho)]=E\left[\int_{0}^{T} \rho_{s-} W_{s-} \mathrm{d}\left(b_{s}-r_{s}\right)\right]
$$

Proof. By the definition of outstanding in (7),

$$
\int_{0}^{T} \rho_{s-} \mathrm{d} W_{s}=-\int_{0}^{T} \rho_{s-} \mathrm{d} A_{s}+\int_{0}^{T} \rho_{s-} W_{s-} \mathrm{d} b_{s}
$$

On the other hand, Lemma 4 and the initial conditions $W_{0}=-A_{0}$ and $\rho_{0}=1$ give

$$
\int_{0}^{T} \rho_{s-} \mathrm{d} W_{s}=\rho_{T} W_{T}+A_{0}-\int_{0}^{T} W_{s-} \mathrm{d} \rho_{s}-\sum_{0<s \leq T} \Delta \rho_{s} \Delta W_{s}
$$

Equating the right-hand sides, we obtain

$$
A_{0}+\int_{0}^{T} \rho_{s-} \mathrm{d} A_{s}=-\rho_{T} W_{T}+\int_{0}^{T} \rho_{s-} W_{s-} \mathrm{d}\left(b_{s}-\tau_{s}\right)+\sum_{0<s \leq T} \Delta \rho_{\mathrm{s}} \Delta W_{s}
$$

and (11) is established. Equations (12) and (13) follow from (11) and Proposition 5. a

\section{Conclusions}

This work has applied to a decomposition method for discounted cash flows firstly proposed by Peccati [7] the simple observation that the theory of semimartingales can be conveniently used to unify the modelization of deterministic and stochastic financial 
operations. In fact, by grouping all the financial operations whose cumulant is a semimartingale in the class of the standard financial operations, we have been able to provide a natural extension of the definitions of discounted cash flow and outstanding, which are crucial for the decomposition method.

Building on these, we have discussed both the construction of the internal financial law for a standard financial operation and the decomposition of its discounted cash flow under the assumption of deterministic discount factors. Successively, we have considered the possibility of random discount factors and we have proposed two alternative ways to define the internal financial law of a standard financial operation, providing decomposition formulas for both of them.

\section{REFERENCES}

[1] F. BECCACECE (1990), «Sulla scomposizione del DCF», in Atti XIV Convegno AMASES, forthcoming.

[2] E. Castagnoli, L. Peccat7 (1973), «Alcune considerazioni in tema di classificazione degli investimenti», Giornale degli Economisti ed Annali di Economia 32; 235-252.

[3] J. GRANDEll (1991), "Aspects of Risk Theory», Springer-Verlag, New York.

[4] H. HOTELLING (1925), «A general mathematical theory of depreciation», Joumal of the American Statistical Association 20, 340-353.

[5] E. LEVI (1964), Corso di matematica finanziaria ed attuariale, Giuffrè, Milano.

[6] E. LUCIANO, L. PECCATI (1990), «The decomposition of random discounted cash flows», presented at the $7^{\text {th }}$ mecting of the EURO Working Group on Financial Modelling, Sirmione, April 5-7.

[7] L. PECCATT (1987), «DCF e risultati di periodo», in Atti XI Convegno AMASES, Bologna, Pitagora Editrice, 483-493.

[8] L. PECCATI (1989), «La valutazione di attività finanziarie singole ed in portafoglio», presented at the Congress I metodi quantitativi per le applicazioni finanziarie, Siena, November 12-14.

[9] L. PECCATT (1991), "Valutazione analitica e sintetica di attività finanziarie», Quademi della Rivista Milancse di Economia, forthcoming.

[10] P. PROTTER (1990), Stochastic integration and differential equations, Springer-Verlag, Berlin.

Sulla scomposizione di risultati

economici attualizzati stocastici

\section{RIASSUNTO}

Il lavoro propone un 'estensione al caso aleatorio dei risultati presentati in Peccati [7] e Beccacece [1] sulla scomposizione del risultato economico attualizzato per operazioni finanziarie certe. Considerando la classe di operazioni finanziarie la cui funzione cumulativa dei margini è una semimartingala, si perviene ad una formula di decomposizione 
del risultato economico attualizzato molto generale, valida anche nel caso di fattori di sconto aleatori. 